\title{
Design Optimization of a Speed Reducer Using Deterministic Techniques
}

\author{
Ming-Hua Lin, ${ }^{1}$ Jung-Fa Tsai, $^{2}$ Nian-Ze Hu, ${ }^{3}$ and Shu-Chuan Chang ${ }^{2,4}$ \\ ${ }^{1}$ Department of Information Technology and Management, Shih Chien University, No. 70, Dazhi Street, Taipei 10462, Taiwan \\ ${ }^{2}$ Department of Business Management, National Taipei University of Technology, No. 1, Section 3, Chung-Hsiao E. Road, \\ Taipei 10608, Taiwan \\ ${ }^{3}$ Department of Information Management, No. 64, Wunhua Road, Huwei Township, Yunlin County 632, Taiwan \\ ${ }^{4}$ Department of Information Management, St. John's University, No. 499, Section 4, Tam King Road, Tamsui District, \\ New Taipei City 25135, Taiwan \\ Correspondence should be addressed to Jung-Fa Tsai; jftsai@ntut.edu.tw
}

Received 26 June 2013; Accepted 10 September 2013

Academic Editor: Yi-Chung Hu

Copyright (c) 2013 Ming-Hua Lin et al. This is an open access article distributed under the Creative Commons Attribution License, which permits unrestricted use, distribution, and reproduction in any medium, provided the original work is properly cited.

\begin{abstract}
The optimal design problem of minimizing the total weight of a speed reducer under constraints is a generalized geometric programming problem. Since the metaheuristic approaches cannot guarantee to find the global optimum of a generalized geometric programming problem, this paper applies an efficient deterministic approach to globally solve speed reducer design problems. The original problem is converted by variable transformations and piecewise linearization techniques. The reformulated problem is a convex mixed-integer nonlinear programming problem solvable to reach an approximate global solution within an acceptable error. Experiment results from solving a practical speed reducer design problem indicate that this study obtains a better solution comparing with the other existing methods.
\end{abstract}

\section{Introduction}

Many engineering design problems are formulated as mathematical programming models. In last few decades, these nonlinear engineering problems have been investigated in much research that solved the formulated problems by different methods. The methods can be generally categorized into metaheuristic and deterministic approaches. To compare the performance of different optimization algorithms, several structural engineering applications are often solved to validate or test the suitability of the optimization algorithms. The speed reducer problem is one of the benchmark problems in structural optimization. The problem represents the design of a simple gear box used in a light airplane between the engine and propeller to allow each to rotate at its most efficient speed.

A large number of algorithms have been developed to solve different engineering optimization problems. In order to overcome the computational drawbacks of existing numerical methods, many metaheuristic algorithms that combine rules and randomness to imitate natural phenomena [1] have been developed. The most general metaheuristic methods include evolutionary computation (EC), tabu search (TS), simulated annealing (SA), ant colony optimization (ACO), and particle swarm (PS) [2]. The surveys of applications and algorithmic advances for metaheuristic algorithms are provided by Glover and Kochenberger [3], Lee and Geem [1], and Bianchi et al. [4]. Li and Papalambros [5] used the global optimization knowledge that is incorporated in several types of rules concerning constraint activity, redundancy, and dominance to solve the speed reducer problem. Ku et al. [6] solved the speed reducer problem using the Taguchi method that emphasizes the design of a robust product insensitive to disturbances. Akhtar et al. [7] developed an optimization algorithm based on a sociobehavioural concept of society and 
civilization to solve the same problem. The essence of their methodology is derived from the concept that the behaviour of an individual changes and improves due to social interaction with the society leaders. Rao and Xiong [8] proposed a hybrid genetic algorithm that combines the advantages of random search and deterministic search methods to improve the convergence speed and computational efficiency for solving mixed-discrete nonlinear design optimization problems. They also solved the speed reducer problem to demonstrate the effectiveness and robustness of their approach. Cagnina et al. [9] developed a particle swarm optimization algorithm to solve constrained engineering optimization problems and used four standard engineering design problems including a speed reducer problem to validate their algorithm. Jaberipour and Khorram [2] proposed two new harmony search (HS) metaheuristic algorithms for engineering optimization problems with continuous design variables and applied their method to solve the speed reducer problem.

Although the metaheuristic algorithms have the advantages of broad applicability, easy implementation, and robustness, these methods cannot guarantee global optimality of the solution. Several deterministic approaches based on mathematical programming techniques have been developed to solve engineering design problems. Tosserams et al. [10] proposed a decomposed problem formulation based on the augmented Lagrangian penalty function and the block coordinate descent algorithm for quasiseparable multidisciplinary design optimization problems. They solved the speed reducer design problem by the proposed decomposition algorithms. $\mathrm{Lu}$ and Kim [11] proposed a decomposition algorithm for the multidisciplinary design optimization problems with complementarity constraints based on the regularization technique and inexact penalty decomposition. They also solved the design problem of a speed reducer. One major deterministic approach to globally solve generalized geometric programming problems is to reformulate the original problems into convex mixed-integer nonlinear programming (MINLP) problems. Some transformation techniques have been developed to convexify the nonconvex functions. Pörn et al. [12] introduced different convexification strategies to deal with posynomial and negative binomial terms. Floudas and Pardalos [13] and Maranas and Floudas [14] proposed exponential transformations to treat nonconvex terms. Lundell et al. [15] proposed some transformation techniques to solve optimization problems including signomial functions to global optimality. $\mathrm{Li}$ and $\mathrm{Lu}$ [16] applied convexification strategies and piecewise linearization techniques to solve generalized geometric programming problems with free discrete/continuous variables. Tsai and Lin [17] proposed an efficient method to solve a posynomial geometric programming problem with separable functions by applying an appropriate variable transformation and an efficient piecewise linearization formulation. Lin et al. [18] used convexification strategies and piecewise linearization techniques to solve engineering optimization problems including the speed reducer design problem. Lu [19] proposed a convexification transformation method (beta method) based on the concept of 1-convex functions to improve the efficiency of solving generalized geometric programming problems. Huang [20] proposed

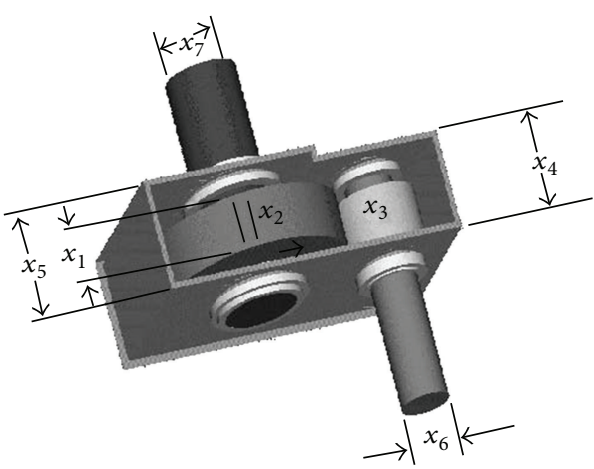

FIGURE 1: Speed reducer design [24].

a deterministic optimization approach to solve geometric programming problems including the speed reducer design problem. His method converts all signomial terms into convex and concave terms, and then the concave terms are further treated with a piecewise linearization method. Lin and Tsai [21] also used convexification strategies and piecewise linearization techniques to solve the speed reducer design problem. This study applies an efficient optimization approach to globally solve speed reducer design problems based on deterministic techniques. In addition to convexification strategies and piecewise linearization techniques, this study applies optimization-based range reduction techniques $[22,23]$ to improve computational efficiency in globally solving the speed reducer design problem. Compared with existing methods, the proposed method is capable of obtaining a better solution.

The rest of the paper is organized as follows. Section 2 describes the process of globally solving a speed reducer design problem. A practical speed reducer problem is solved in Section 3 to demonstrate the effectiveness of the proposed method. After that, conclusion remarks are made in Section 4.

\section{Global Optimization Approach of a Speed Reducer Design Problem}

A speed reducer is part of the gear box of mechanical system, and it is used in many other types of applications. The design of the speed reducer is a more challenging benchmark, because it involves seven design variables [25]. As shown in Figure 1, the design of the speed reducer is considered with the face width $\left(x_{1}\right)$, the module of the teeth $\left(x_{2}\right)$, the number of teeth on pinion $\left(x_{3}\right)$, the length of the first shaft between bearings $\left(x_{4}\right)$, the length of the second shaft between bearings $\left(x_{5}\right)$, diameter of the first shaft $\left(x_{6}\right)$, and the diameter of the second shaft $\left(x_{7}\right)$. Another schematic of the speed reducer is presented in Figure 2 with its design variables being labeled.

This problem is taken from Golinski [26]. The objective is to minimize the total weight of the speed reducer while satisfying eleven constraints. The constraints include the limits on the bending stress of the gear teeth, surface stress, transverse deflections of shafts 1 and 2 due to transmitted force, and stresses in shafts 1 and 2. The mathematical 


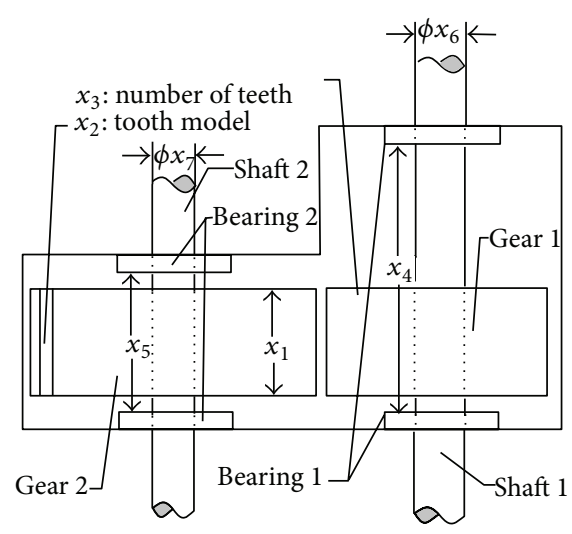

FIgURE 2: A schematic of the speed reducer [11].

programming model of a speed reducer problem considered in this study is expressed as follows.

$$
\begin{aligned}
& \text { Minimize } f\left(x_{1}, \ldots, x_{7}\right) \\
& =0.7854 x_{1} x_{2}^{2} \\
& \times\left(3.3333 x_{3}^{2}+14.9334 x_{3}-43.0934\right) \\
& -1.508 x_{1}\left(x_{6}^{2}+x_{7}^{2}\right)+7.4777\left(x_{6}^{3}+x_{7}^{3}\right) \\
& +0.7854\left(x_{4} x_{6}^{2}+x_{5} x_{7}^{2}\right) \text {, } \\
& \text { subject to } g_{1}=\frac{27}{x_{1} x_{2}^{2} x_{3}}-1 \leq 0 \text {, } \\
& g_{2}=\frac{397.5}{x_{1} x_{2}^{2} x_{3}^{2}}-1 \leq 0 \text {, } \\
& g_{3}=\frac{1.93 x_{4}^{3}}{x_{2} x_{3} x_{6}^{4}}-1 \leq 0 \text {, } \\
& g_{4}=\frac{1.93 x_{5}^{3}}{x_{2} x_{3} x_{7}^{4}}-1 \leq 0 \text {, } \\
& g_{5}=\frac{1}{110 x_{6}^{3}} \sqrt{\left(\frac{745 x_{4}}{x_{2} x_{3}}\right)^{2}+16.9 \times 10^{6}}-1 \\
& \leq 0 \text {, } \\
& g_{6}=\frac{1}{85 x_{7}^{3}} \sqrt{\left(\frac{745 x_{5}}{x_{2} x_{3}}\right)^{2}+157.5 \times 10^{6}}-1 \\
& \leq 0 \text {, } \\
& g_{7}=\frac{x_{2} x_{3}}{40}-1 \leq 0 \\
& g_{8}=\frac{5 x_{2}}{x_{1}}-1 \leq 0, \\
& g_{9}=\frac{x_{1}}{12 x_{2}}-1 \leq 0 \text {, }
\end{aligned}
$$

$$
\begin{aligned}
& g_{10}=\frac{1.5 x_{6}+1.9}{x_{4}}-1 \leq 0, \\
& g_{11}=\frac{1.1 x_{7}+1.9}{x_{5}}-1 \leq 0, \\
& 2.6 \leq x_{1} \leq 3.6, \quad 0.7 \leq x_{2} \leq 0.8, \\
& 17 \leq x_{3} \leq 28, \quad 7.3 \leq x_{4} \leq 8.3, \\
& 7.3 \leq x_{5} \leq 8.3, \\
& 5 \leq x_{7} \leq 5.5 .
\end{aligned}
$$

The original speed reducer problem described previously can be simplified as the following generalized geometric programming problem.

$$
\text { Minimize } \begin{aligned}
& f\left(x_{1}, \ldots, x_{7}\right) \\
&=(0.7854 \times 3.3333) x_{1} x_{2}^{2} x_{3}^{2} \\
&+(0.7854 \times 14.9334) x_{1} x_{2}^{2} x_{3} \\
&-(0.7854 \times 43.0934) x_{1} x_{2}^{2} \\
&-1.508 x_{1} x_{6}^{2}-1.508 x_{1} x_{7}^{2}+7.4777 x_{6}^{3} \\
&+7.4777 x_{7}^{3}+0.7854 x_{4} x_{6}^{2}+0.7854 x_{5} x_{7}^{2}, \\
& \text { subject to } g_{1}= 27 x_{1}^{-1} x_{2}^{-2} x_{3}^{-1}-1 \leq 0, \\
& g_{2}= 397.5 x_{1}^{-1} x_{2}^{-2} x_{3}^{-2}-1 \leq 0, \\
& g_{3}= 1.93 x_{4}^{3} x_{2}^{-1} x_{3}^{-1} x_{6}^{-4}-1 \leq 0, \\
& g_{4}= 1.93 x_{5}^{3} x_{2}^{-1} x_{3}^{-1} x_{7}^{-4}-1 \leq 0, \\
& g_{5}= 745^{2} x_{4}^{2} x_{2}^{-2} x_{3}^{-2}-110^{2} x_{6}^{6}+16.9 \times 10^{6} \\
& \leq 0, \\
& g_{6}= 745^{2} x_{5}^{2} x_{2}^{-2} x_{3}^{-2}-85^{2} x_{7}^{6}+157.5 \times 10^{6} \\
& \leq 0, \\
& g_{7}= x_{2} x_{3}-40 \leq 0, \\
& g_{8}= 5 x_{2}-x_{1} \leq 0, \\
& g_{9}= x_{1}-12 x_{2} \leq 0, \\
& g_{10}= 1.5 x_{6}-x_{4}+1.9 \leq 0, \\
& g_{11}= 1.1 x_{7}-x_{5}+1.9 \leq 0, \\
& 2.6 \leq x_{1} \leq 3.6, \\
& 17 \leq x_{3} \leq 28, \\
& 7.3 \leq x_{5} \leq 8.3, \\
& 5 \leq x_{7} \leq 5.5 . \\
& \\
& \\
&
\end{aligned}
$$

The simplified problem above is a nonconvex program. Based on the deterministic techniques, this study transforms the problem into a convex MINLP problem by the convexification strategies and piecewise linearization methods. Then 
the reformulated problem can be solved by convex MINLP solvers to obtain a global optimal solution.

First, we determine that certain classes of signomial terms in the above simplified problem are convex and do not necessitate any transformations. Consequently, the number of concave functions requiring to be piecewise linearized decreases, and the resulting problem is a computationally efficient model. $x_{6}^{3}$ and $x_{7}^{3}$ are convex terms. According to Maranas and Floudas [27], $x_{1}^{-1} x_{2}^{-2} x_{3}^{-1}$ and $x_{1}^{-1} x_{2}^{-2} x_{3}^{-2}$ are also convex terms. Then, the nonconvex monomials are transformed. By taking exponential transformation [28, 29] on the variable with a positive exponent, the positive monomial terms $x_{1} x_{2}^{2} x_{3}^{2}, x_{1} x_{2}^{2} x_{3}, x_{4} x_{6}^{2}, x_{5} x_{7}^{2}, x_{4}^{3} x_{2}^{-1} x_{3}^{-1} x_{6}^{-4}$, $x_{5}^{3} x_{2}^{-1} x_{3}^{-1} x_{7}^{-4}, x_{4}^{2} x_{2}^{-2} x_{3}^{-2}, x_{5}^{2} x_{2}^{-2} x_{3}^{-2}$, and $x_{2} x_{3}$ are transformed into convex terms $e^{y_{1}+2 y_{2}+2 y_{3}}, e^{y_{1}+2 y_{2}+y_{3}}, e^{y_{4}+2 y_{6}}, e^{y_{5}+2 y_{7}}$, $e^{3 y_{4}} x_{2}^{-1} x_{3}^{-1} x_{6}^{-4}, e^{3 y_{5}} x_{2}^{-1} x_{3}^{-1} x_{7}^{-4}, e^{2 y_{4}} x_{2}^{-2} x_{3}^{-2}, e^{2 y_{5}} x_{2}^{-2} x_{3}^{-2}$, and $e^{y_{2}+y_{3}}$, respectively, where $y_{i}=\ln x_{i}, i=1,2, \ldots, 7$. By taking power transformations [28-32] on the variables to make the sum of the exponents not greater than one, the negative monomial terms $-x_{1} x_{2}^{2},-x_{1} x_{6}^{2},-x_{1} x_{7}^{2},-x_{6}^{6}$, and $-x_{7}^{6}$ are transformed into convex terms $-z_{1}^{1 / 3} z_{2}^{2 / 3},-z_{1}^{1 / 3} z_{6}^{1 / 3}$, $-z_{1}^{1 / 3} z_{7}^{1 / 3},-z_{6}$, and $-z_{7}$, respectively, where $z_{i}=x_{i}^{3}, i=1,2$, $z_{i}=x_{i}^{6}, i=6,7$.

The nonconvex problem can be convexified and underestimated by the convexification strategies mentioned previously if the inverse transformations $\left(y_{i}=\ln x_{i}, i=\right.$ $1,2, \ldots, 7, z_{i}=x_{i}^{3}, i=1,2$, and $\left.z_{i}=x_{i}^{6}, i=6,7\right)$ are approximated by piecewise linear functions. The efficiency of the piecewise linearization technique has a critical impact on the computational efficiency in solving the reformulated problems. Vielma and Nemhauser [33] proposed a linearization approach that has favorable tightness properties. Their experimental results showed that the Vielma and Nemhauser [33] method significantly outperforms other models. Tsai and Lin [17] employed the Vielma and Nemhauser [33] method to solve posynomial geometric programming problems. This study also adopts the Vielma and Nemhauser [33] method to linearly approximate the inverse transformations. Compared with the Lin and Tsai [21] method, this study utilizes range reduction techniques $[22,23]$ to further improve the computational efficiency.

Adding more break points can construct a tighter underestimator of the original problem, and the obtained solution is more closer to the real global solution. If $g_{i}(x)<0$ is the $i$ th constraint and $x^{*}$ is the solution derived from the reformulated model, then the number of break points does not need to increase until $\operatorname{Max}_{i}\left(g_{i}\left(x^{*}\right)\right) \leq \varepsilon$, where $\varepsilon$ is the feasibility tolerance.

\section{Computational Experiments}

By using the deterministic approach introduced above, this study reformulates the original speed reducer problem as a convex MINLP problem as follows.

$$
\begin{aligned}
\text { Minimize } \quad f & \left(x_{1}, \ldots, x_{7}\right) \\
& =(0.7854 \times 3.3333) e^{y_{1}+2 y_{2}+2 y_{3}}
\end{aligned}
$$

$$
\begin{aligned}
& +(0.7854 \times 14.9334) e^{y_{1}+2 y_{2}+y_{3}} \\
& -(0.7854 \times 43.0934) z_{1}^{1 / 3} z_{2}^{2 / 3} \\
& -1.508 z_{1}^{1 / 3} z_{6}^{1 / 3}-1.508 z_{1}^{1 / 3} z_{7}^{1 / 3} \\
& +7.4777 x_{6}^{3}+7.4777 x_{7}^{3} \\
& +0.7854 e^{y_{4}+2 y_{6}}+0.7854 e^{y_{5}+2 y_{7}}
\end{aligned}
$$

subject to $g_{1}=27 x_{1}^{-1} x_{2}^{-2} x_{3}^{-1}-1 \leq 0$,

$$
g_{2}=397.5 x_{1}^{-1} x_{2}^{-2} x_{3}^{-2}-1 \leq 0 \text {, }
$$$$
g_{3}=1.93 e^{3 y_{4}} x_{2}^{-1} x_{3}^{-1} x_{6}^{-4}-1 \leq 0 \text {, }
$$$$
g_{4}=1.93 e^{3 y_{5}} x_{2}^{-1} x_{3}^{-1} x_{7}^{-4}-1 \leq 0,
$$$$
g_{5}=745^{2} e^{2 y_{4}} x_{2}^{-2} x_{3}^{-2}-110^{2} z_{6}+16.9 \times 10^{6}
$$$$
\leq 0 \text {, }
$$$$
g_{6}=745^{2} e^{2 y_{5}} x_{2}^{-2} x_{3}^{-2}-85^{2} z_{7}+157.5 \times 10^{6}
$$$$
\leq 0 \text {, }
$$$$
g_{7}=e^{y_{2}+y_{3}}-40 \leq 0 \text {, }
$$$$
g_{8}=5 x_{2}-x_{1} \leq 0,
$$$$
g_{9}=x_{1}-12 x_{2} \leq 0 \text {, }
$$$$
g_{10}=1.5 x_{6}-x_{4}+1.9 \leq 0 \text {, }
$$$$
g_{11}=1.1 x_{7}-x_{5}+1.9 \leq 0,
$$$$
y_{i}=L\left(\ln x_{i}\right), \quad i=1,2, \ldots, 7,
$$$$
z_{i}=L\left(x_{i}^{3}\right), \quad i=1,2,
$$$$
z_{i}=L\left(x_{i}^{6}\right), \quad i=6,7,
$$$$
2.6 \leq x_{1} \leq 3.6, \quad 0.7 \leq x_{2} \leq 0.8,
$$$$
17 \leq x_{3} \leq 28, \quad 7.3 \leq x_{4} \leq 8.3,
$$$$
7.3 \leq x_{5} \leq 8.3, \quad 2.9 \leq x_{6} \leq 3.9
$$$$
5 \leq x_{7} \leq 5.5 \text {. }
$$

In the transformation process, the piecewise linearization technique introduced by Vielma and Nemhauser [33] is utilized to approximate $\ln x_{i}(i=1,2, \ldots, 7), x_{i}^{3}(i=1,2)$, and $x_{i}^{6}(i=6,7)$. The Vielma and Nemhauser [33] method represents a piecewise linear function with $m$ break points by $\left\lceil\log _{2} m\right\rceil$ binary variables. By using $3,4, \ldots, 9$ binary variables, respectively, we convert this program to a convex MINLP problem with $8,16, \ldots, 512$ break points, respectively, used in linearly approximating the inverse transformations. The reformulated problems are solved by LINGO [34]. Table 1 lists the reported solutions from LINGO, objective values on 
TABLE 1: Experiment results of the speed reducer problem by the proposed method under different numbers of break points.

\begin{tabular}{lccccc}
\hline $\begin{array}{l}\text { No. of break } \\
\text { points }\end{array}$ & $\begin{array}{c}\text { No. of binary } \\
\text { variables }\end{array}$ & Reported solution $\left(x_{1}, x_{2}, x_{3}, x_{4}, x_{5}, x_{6}, x_{7}\right)$ & $\begin{array}{c}\text { CPU time } \\
(\mathrm{mm}: \mathrm{ss})\end{array}$ & $\begin{array}{c}\text { Objective } \\
\text { value }\end{array}$ & $\begin{array}{c}\text { Error in } \\
\text { constraint }\end{array}$ \\
\hline 8 & 3 & $(3.5,0.7,17.0,7.3,7.714826,3.347398,5.286205)$ & $00: 07$ & 2993.457535 & 0.002526473 \\
16 & 4 & $(3.5,0.7,17.0,7.3,7.715248,3.349748,5.286589)$ & $00: 09$ & 2994.308994 & 0.000418000 \\
32 & 5 & $(3.5,0.7,17.0,7.3,7.715291,3.350039,5.286628)$ & $00: 10$ & 2994.408856 & 0.000157319 \\
64 & 6 & $(3.5,0.7,17.0,7.3,7.715313,3.350187,5.286648)$ & $00: 42$ & 2994.459757 & 0.000024774 \\
128 & 7 & $(3.5,0.7,17.0,7.3,7.715318,3.353125,5.286653)$ & $01: 27$ & 2994.467375 & 0.000009551 \\
256 & 8 & $(3.5,0.7,17.0,7.3,7.715320,3.350213,5.286654)$ & $05: 23$ & 2994.470348 & 0.000001492 \\
512 & 9 & $(3.5,0.7,17.0,7.3,7.715320,3.350214,5.286654)$ & $15: 09$ & 2994.470603 & 0.000000596 \\
\hline
\end{tabular}

TABLE 2: Experiment results of the speed reducer problem by the proposed method with range reduction.

\begin{tabular}{|c|c|c|c|c|c|}
\hline Iteration & Variable bound & Reported solution $\left(x_{1}, x_{2}, x_{3}, x_{4}, x_{5}, x_{6}, x_{7}\right)$ & $\begin{array}{l}\text { Accumulated CPU } \\
\text { time (mm:ss) }\end{array}$ & $\begin{array}{l}\text { Objective } \\
\text { value }\end{array}$ & $\begin{array}{l}\text { Error in } \\
\text { constraint }\end{array}$ \\
\hline 1 & $\begin{array}{c}{[2.6,3.6]} \\
{[0.7,0.8]} \\
{[17,28]} \\
{[7.3,8.3]} \\
{[7.3,8.3]} \\
{[2.9,3.9]} \\
{[5,5.5]}\end{array}$ & $(3.5,0.7,17.0,7.3,7.715291,3.350039,5.286628)$ & 00:10 & 2994.408856 & 0.000157319 \\
\hline 2 & $\begin{array}{c}{[3.5,3.500182]} \\
{[0.7,0.700011]} \\
{[17,17.000419]} \\
{[7.3,7.307746]} \\
{[7.715291,7.718508]} \\
{[3.350039,3.350290]} \\
{[5.286628,5.286654]}\end{array}$ & $(3.5,0.7,17.0,7.3,7.7153190,3.350282,5.286654)$ & 07:56 & 2994.471921 & 0.000000264 \\
\hline
\end{tabular}

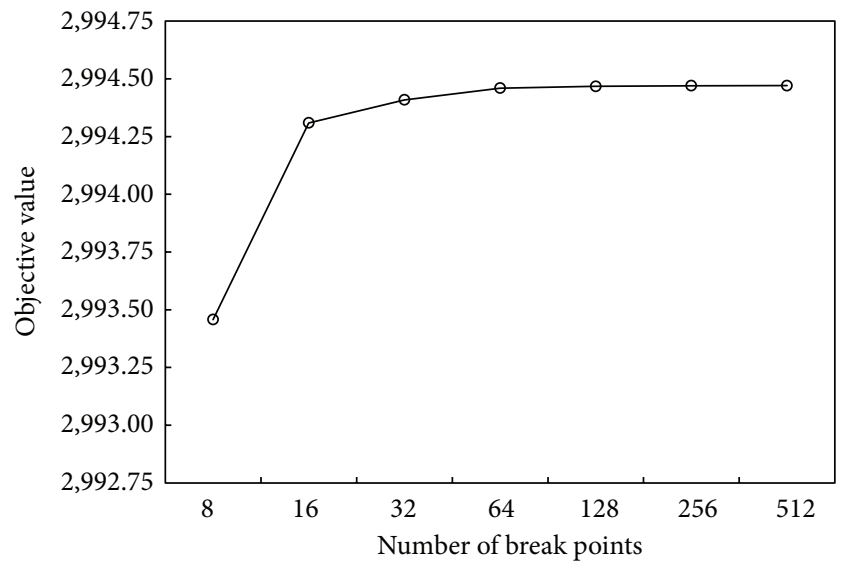

FIGURE 3: Objective values of the speed reducer problem under different numbers of break points.

the reported solutions, and errors in constraint $\operatorname{Max}_{i}\left(g_{i}\left(x^{*}\right)\right)$ under different numbers of break points. Using more break points derives a solution with a lower error in constraint. Figure 3 indicates the objective value obtained from the proposed method under different numbers of break points. We observe that the objective value approximates the real global objective value better as the number of break points increases. Figure 4 indicates the CPU time required to solve the speed reducer problem under different numbers of break points. The required CPU time to solve the reformulated model tends to grow exponentially as the number of break points becomes large. To enhance computational efficiency, this study applies the range reduction techniques to effectively tighten variable bounds. Table 2 lists variable bound, solution, objective value, accumulated CPU time, and error in constraint in each iteration to solve this problem by the proposed method with range reduction. Thirty-two break points are used in the piecewise linearization process in each iteration. The accumulated CPU time consists of the CPU time to update variable bounds and solve the reformulated models iteratively. The global solution (3.5, 0.7, 17.0, 7.3, $7.7153190,3.350282$, and 5.286654) with objective 2994.471921 and an error in constraint below $10^{-6}$ can be obtained within 8 minutes. If no range reduction is adopted and 512 line segments are used in the piecewise linearization process, the global solution with an error in constraint below $10^{-6}$ is obtained within 16 minutes as shown in Table 1.

Table 3 displays the comparison of results with existing methods. The solutions listed in the table are reported from their original research, and the objective values are computed from the objective function $f\left(x_{1}, \ldots, x_{7}\right)=$ $0.7854 x_{1} x_{2}^{2}\left(3.3333 x_{3}^{2}+14.9334 x_{3}-43.0934\right)-1.508 x_{1}\left(x_{6}^{2}+x_{7}^{2}\right)$ $+7.4777\left(x_{6}^{3}+x_{7}^{3}\right)+0.7854\left(x_{4} x_{6}^{2}+x_{5} x_{7}^{2}\right)$ on the reported solutions. Tosserams et al. [10] and Lu and Kim [11] obtained 
TABLE 3: Comparisons of optimal solutions of the speed reducer problem by different methods.

\begin{tabular}{|c|c|c|c|c|}
\hline Methods & Method type & $\begin{array}{c}\text { Reported solution } \\
\left(x_{1}, x_{2}, x_{3}, x_{4}, x_{5}, x_{6}, x_{7}\right)\end{array}$ & Objective value & $\begin{array}{c}\text { Error in } \\
\text { constraint }\end{array}$ \\
\hline Ku et al. [6] & Metaheuristic & $(3.6,0.7,17,7.3,7.8,3.4,5.0)$ & 2876.219475 & $<10^{0}$ \\
\hline Akhtar et al. [7] & Metaheuristic & $\begin{array}{c}(3.506122,0.700006,17,7.549126 \\
\text { 7.859330, 3.365576, 5.289773) }\end{array}$ & 3008.197440 & $<10^{-6}$ \\
\hline Rao and Xiong [8] & Metaheuristic & $(3.5,0.7,17,7.3,7.8,3.36,5.29)$ & 3000.959715 & $<10^{-6}$ \\
\hline Cagnina et al. [9] & Metaheuristic & $(3.5,0.7,17,7.3,7.8,3.350214,5.286683)$ & 2996.347849 & $<10^{-6}$ \\
\hline Jaberipour and Khorram [2] & Metaheuristic & $\begin{array}{c}(3.5,0.7,17,7.3,7.71533233833903 \\
3.35021510925684,5.28666403545462)\end{array}$ & 2994.477531 & $<10^{-6}$ \\
\hline Li and Papalambros [5] & Metaheuristic & $\begin{array}{c}(3.5,0.7,17,7.299999,7.715317,3.350541, \\
5.286654)\end{array}$ & 2994.553869 & $<10^{-6}$ \\
\hline Tosserams et al. [10] & Deterministic & $(3.5,0.7,17,7.3,7.72,3.35,5.29)$ & 2996.645783 & $<10^{-3}$ \\
\hline Lu and Kim [11] & Deterministic & $\begin{array}{c}(3.5,0.7,17,7.3,7.670396,3.542421, \\
5.245814)\end{array}$ & 3019.583365 & $<10^{-1}$ \\
\hline Lin et al. [18] & Deterministic & $(3.5,0.7,17,7.3,7.715,3.349,5.286)$ & 2993.738921 & $<10^{-2}$ \\
\hline Huang [20] & Deterministic & $\begin{array}{c}(3.495652,0.7000002,17,7.30000007 \\
\quad 7.7120386,3.343372,5.285352)\end{array}$ & 2990.124384 & $<10^{-2}$ \\
\hline Proposed method & Deterministic & $\begin{array}{c}(3.5,0.7,17.0,7.3,7.7153190,3.350282 \\
5.286654)\end{array}$ & 2994.471921 & $<10^{-6}$ \\
\hline
\end{tabular}

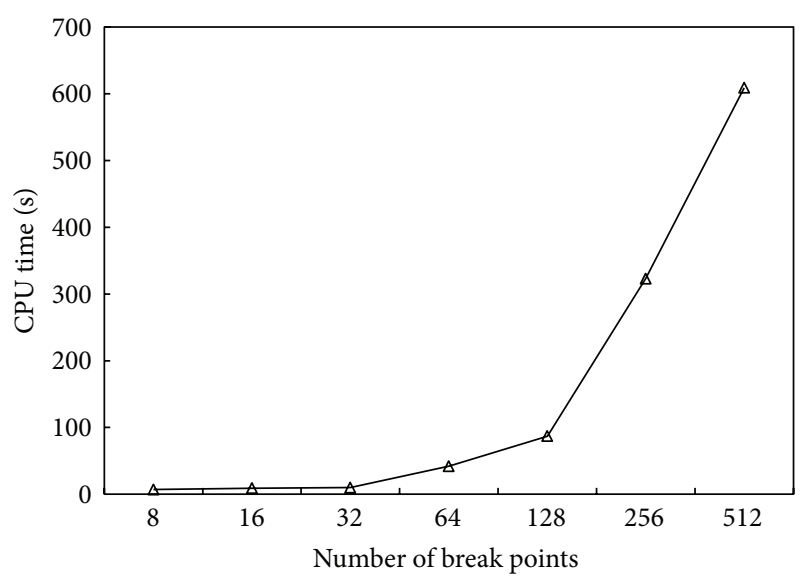

FIGURE 4: CPU time required to solve the speed reducer problem under different numbers of break points.

optimal solutions with higher errors and higher objective values than those of the proposed method. Although Ku et al. [6], Lin et al. [18], and Huang [20] obtained optimal solutions with lower objective values than those of the proposed method, the errors in constraint in these three methods are higher than those in the proposed method. Compared with the solutions of Akhtar et al. [7], Rao and Xiong [8], Cagnina et al. [9], Jaberipour and Khorram [2], and Li and Papalambros [5], the proposed method results in a lower objective value under the same feasibility tolerance $10^{-6}$.

\section{Conclusions}

The speed reducer problem is used in many other types of applications and is one of the standard benchmark problems in structural optimization. Although many metaheuristic algorithms have been developed to solve this problem, these methods cannot guarantee global optimality of the solution. This study applies a deterministic approach based on convexification strategies and piecewise linearization methods to globally solve a speed reducer design problem. However, numerous break points are utilized in the linearization process for reaching an approximate global solution with a low error, and much CPU time is required to solve the reformulated model. Therefore, this study also adopts the range reduction technique to enhance the computational efficiency. Compared with metaheuristic methods, this study guarantees the global optimality of the solution. Compared with other deterministic methods, this study obtains a better solution with a lower error in constraint.

\section{Acknowledgment}

The research is supported by the Taiwan NSC Grants NSC 1012410-H-158-002-MY2 and NSC 102-2410-H-027-012-MY3, and Shih Chien University Grant 102-05-04002.

\section{References}

[1] K. S. Lee and Z. W. Geem, "A new meta-heuristic algorithm for continuous engineering optimization: harmony search theory and practice," Computer Methods in Applied Mechanics and Engineering, vol. 194, no. 36-38, pp. 3902-3933, 2005.

[2] M. Jaberipour and E. Khorram, "Two improved harmony search algorithms for solving engineering optimization problems," Communications in Nonlinear Science and Numerical Simulation, vol. 15, no. 11, pp. 3316-3331, 2010.

[3] F. W. Glover and G. A. Kochenberger, Handbook of Metaheuristics, International Series in Operations Research \& Management Science, Kluwer Academic Publishers, Boston, Mass, USA, 2003. 
[4] L. Bianchi, M. Dorigo, L. M. Gambardella, and W. J. Gutjahr, "A survey on metaheuristics for stochastic combinatorial optimization," Natural Computing, vol. 8, no. 2, pp. 239-287, 2009.

[5] H. L. Li and P. Papalambros, "A production system for use of global optimization knowledge," Journal of Mechanisms, Transmissions, and Automation in Design, vol. 107, no. 2, pp. 277-284, 1985.

[6] K. J. Ku, S. S. Rao, and L. Chen, "Taguchi-aided search method for design optimization of engineering systems," Engineering Optimization, vol. 30, no. 1, pp. 1-23, 1998.

[7] S. Akhtar, K. Tai, and T. Ray, "A socio-behavioral simulation model for engineering design optimization," Engineering Optimization, vol. 34, no. 4, pp. 341-354, 2002.

[8] S. S. Rao and Y. Xiong, "A hybrid genetic algorithm for mixeddiscrete design optimization," Journal of Mechanical Design, vol. 127, no. 6, pp. 1100-1112, 2005.

[9] L. C. Cagnina, S. C. Esquivel, and C. A. Coello Coello, "Solving engineering optimization problems with the simple constrained particle swarm optimizer," Informatica, vol. 32, no. 3, pp. 319326, 2008.

[10] S. Tosserams, L. F. P. Etman, and J. E. Rooda, "An augmented Lagrangian decomposition method for quasi-separable problems in MDO," Structural and Multidisciplinary Optimization, vol. 34, no. 3, pp. 211-227, 2007.

[11] S. Lu and H. M. Kim, "A regularized inexact penalty decomposition algorithm for multidisciplinary design optimization problems with complementarity constraints," Journal of Mechanical Design, vol. 132, no. 4, Article ID 041005, 12 pages, 2010.

[12] R. Pörn, I. Harjunkoski, and T. Westerlund, "Convexification of different classes of non-convex MINLP problems," Computers and Chemical Engineering, vol. 23, no. 3, pp. 439-448, 1999.

[13] C. A. Floudas and P. M. Pardalos, State of the Art in Global Optimization: Computational Methods and Applications, vol. 7 of Nonconvex Optimization and Its Applications, Kluwer Academic Publishers, Dordrecht, The Netherlands, 1996.

[14] C. D. Maranas and C. A. Floudas, "Global optimization in generalized geometric programming," Computers and Chemical Engineering, vol. 21, no. 4, pp. 351-369, 1997.

[15] A. Lundell, J. Westerlund, and T. Westerlund, "Some transformation techniques with applications in global optimization," Journal of Global Optimization, vol. 43, no. 2-3, pp. 391-405, 2009.

[16] H.-L. Li and H.-C. Lu, "Global optimization for generalized geometric programs with mixed free-sign variables," Operations Research, vol. 57, no. 3, pp. 701-713, 2009.

[17] J.-F. Tsai and M.-H. Lin, "An efficient global approach for posynomial geometric programming problems," INFORMS Journal on Computing, vol. 23, no. 3, pp. 483-492, 2011.

[18] M.-H. Lin, J.-F. Tsai, and P.-C. Wang, "Solving engineering optimization problems by a deterministic global optimization approach," Applied Mathematics \& Information Sciences, vol. 6, no. 3, supplement, pp. 1101-1107, 2012.

[19] H.-C. Lu, "An efficient convexification method for solving generalized geometric problems," Journal of Industrial and Management Optimization, vol. 8, no. 2, pp. 429-455, 2012.

[20] C. H. Huang, "Engineering design by geometric programming," Mathematical Problems in Engineering, vol. 2013, Article ID 568098, 8 pages, 2013.

[21] M. H. Lin and J. F. Tsai, "Optimal design of a speed reducer," Applied Mechanics and Materials, vol. 376, pp. 327-330, 2013.
[22] M.-H. Lin and J.-F. Tsai, "Range reduction techniques for improving computational efficiency in global optimization of signomial geometric programming problems," European Journal of Operational Research, vol. 216, no. 1, pp. 17-25, 2012.

[23] C. S. Adjiman, I. P. Androulakis, and C. A. Floudas, "Global optimization of mixedinteger nonlinear problems," AIChE Journal, vol. 46, no. 9, pp. 1769-1797, 2000.

[24] R. Hassan, B. Cohanim, O. de Weck, and G. Venter, "A comparison of particle swarm optimization and the genetic algorithm," in Proceedings of the 1st AIAA Multidisciplinary Design Optimization Specialist Conference, pp. 18-21, April 2005.

[25] A. H. Gandomi and X.-S. Yang, "Benchmark problems in structural optimization," in Computational Optimization, Methods and Algorithms, vol. 356 of Studies in Computational Intelligence, pp. 259-281, Springer, Berlin, Germany, 2011.

[26] J. Golinski, "Optimal synthesis problems solved by means of nonlinear programming and random methods," Journal of Mechanisms, vol. 5, no. 3, pp. 287-309, 1970.

[27] C. D. Maranas and C. A. Floudas, "Finding all solutions of nonlinearly constrained systems of equations," Journal of Global Optimization, vol. 7, no. 2, pp. 143-182, 1995.

[28] R. Pörn, K.-M. Björk, and T. Westerlund, "Global solution of optimization problems with signomial parts," Discrete Optimization, vol. 5, no. 1, pp. 108-120, 2008.

[29] A. Lundell and T. Westerlund, "Convex underestimation strategies for signomial functions," Optimization Methods and Software, vol. 24, no. 4-5, pp. 505-522, 2009.

[30] T. Westerlund, "Some transformation techniques in global optimization," in Global Optimization: From Theory to Implementation, L. Liberti and N. Maculan, Eds., vol. 84, pp. 45-74, Springer, New York, NY, USA, 2007.

[31] J.-F. Tsai and M.-H. Lin, "Global optimization of signomial mixed-integer nonlinear programming problems with free variables," Journal of Global Optimization, vol. 42, no. 1, pp. 3949, 2008.

[32] A. Lundell, J. Westerlund, and T. Westerlund, "Some transformation techniques with applications in global optimization," Journal of Global Optimization, vol. 43, no. 2-3, pp. 391-405, 2009.

[33] J. P. Vielma and G. L. Nemhauser, "Modeling disjunctive constraints with a logarithmic number of binary variables and constraints," Mathematical Programming, vol. 128, no. 1-2, pp. 49-72, 2011.

[34] LINGO, Release. 11.0, Lindo System Inc., Chicago, Ill, USA, 2004. 


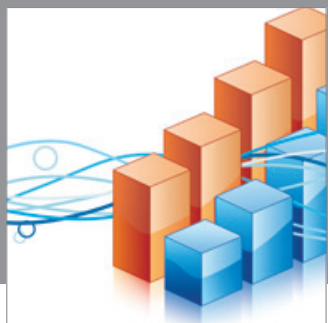

Advances in

Operations Research

mansans

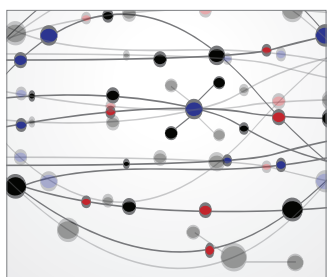

The Scientific World Journal
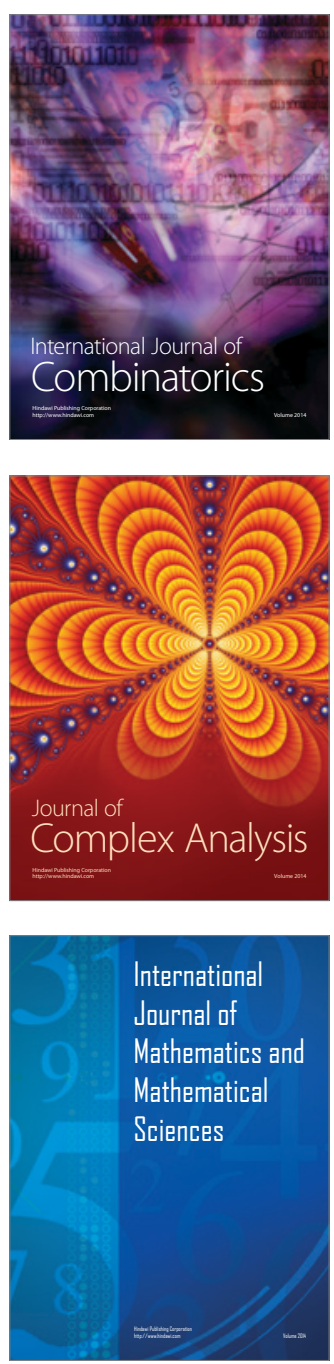
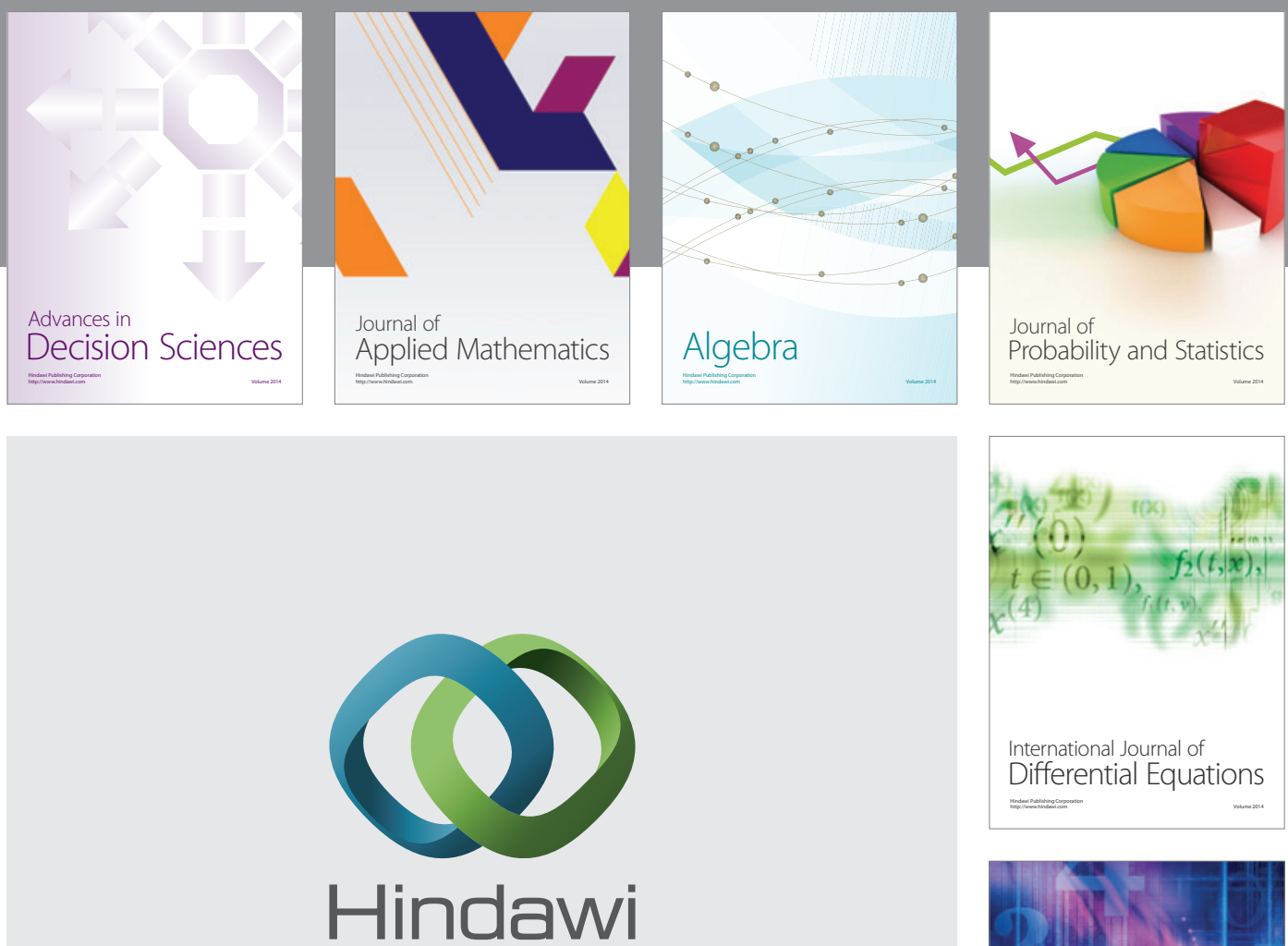

Submit your manuscripts at http://www.hindawi.com
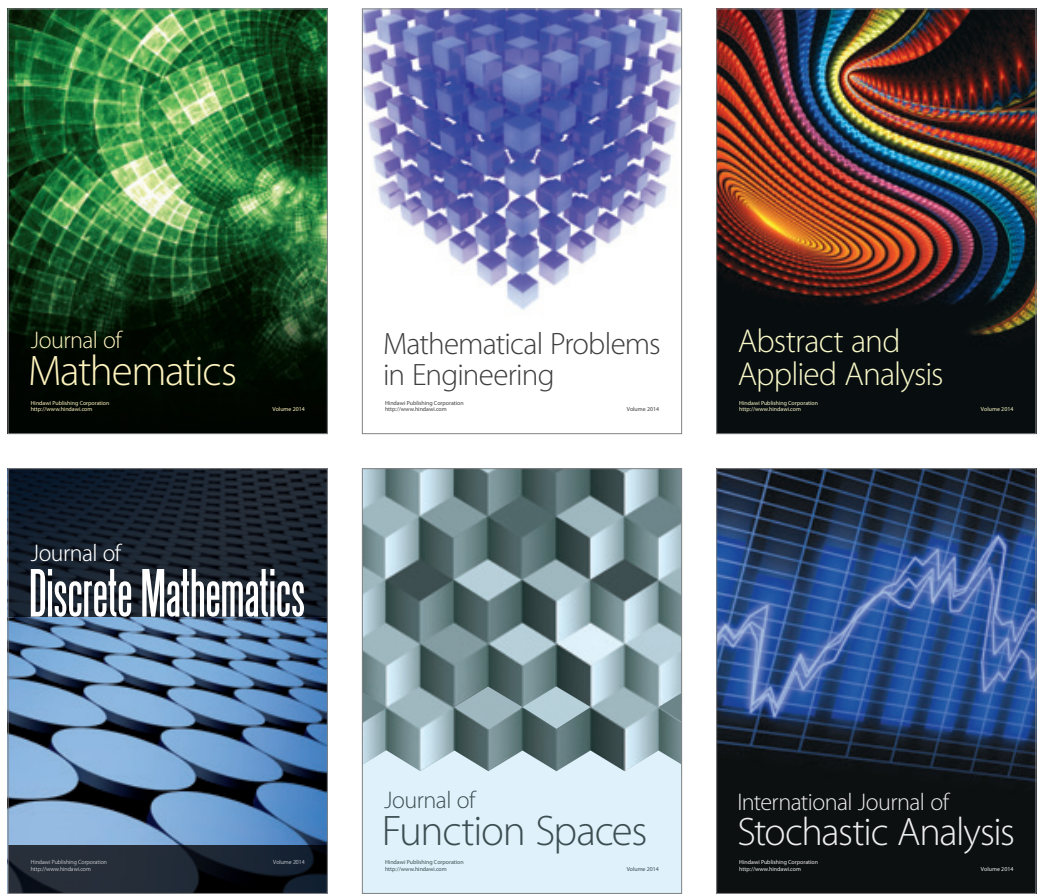

Journal of

Function Spaces

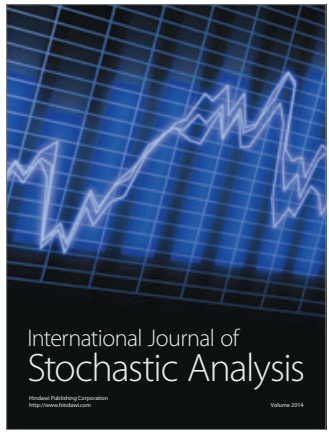

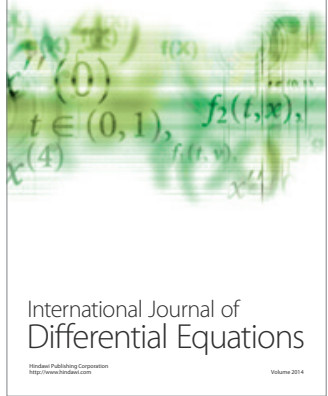
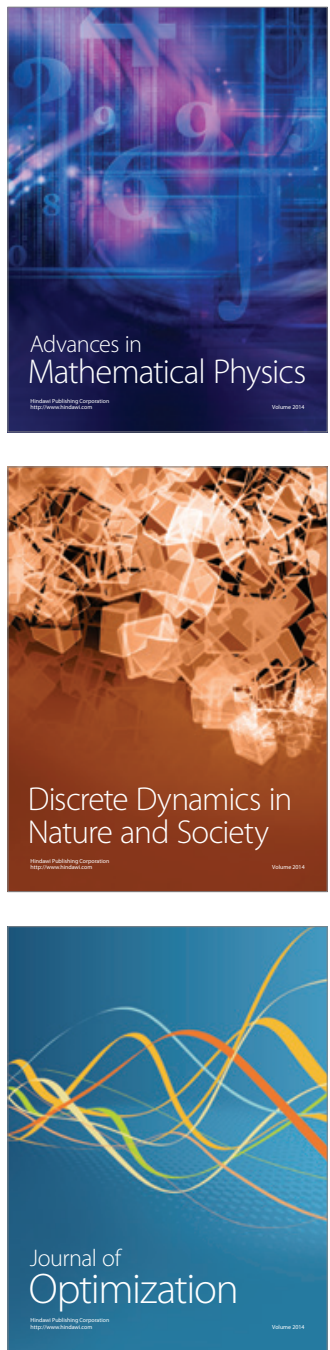